\section{POPULATION-BASED SHAPE OPTIMIZATION OF A CENTRIFUGAL PUMP IMPELLER}

PROKOP MORAVEC, PAVEL RUDOLF

Brno University of Technology, Faculty of Mechanical Engineering, V. Kaplan Department of Fluid Engineering

DOI : 10.17973/MMSJ.2020_10_2020019

115861@vutbr.cz

A shape optimization problem is increasingly popular in various parts of industry and academia. Several shape optimization approaches emerged in the past few years. Population-based methods (PPM) are among them, with convenient attributes and characteristics that lead to a discovery of proper solutions of investigated problems. Crucial quality of PPM lies primarily in number of randomly scattered individuals, which can move through a whole given computational area.

The chosen PPM in this research of a finding of a proper design of the centrifugal pump impeller is Particle Swarm Optimization Algorithm (shortly PSOA). This algorithm is strongly influenced by a social behaviour of various animals, such as ants or fish. Each member of the swarm moves in the whole given computational space and is strongly attracted to an individual with the best value of the examined function, e.g. pump efficiency.

The research focuses on a new tool for automatic shape optimization based on the Multi-objective PSO and its outcome - three optimized designs of the centrifugal pump impeller. These three impellers are compared on the performance characteristics basis. The essential differences are discussed, and the design of a suitable impeller is outlined.

KEYWORDS

shape optimization, multi-objective optimization, centrifugal pump, pump impeller, Particle Swarm Optimization, CFD

\section{INTRODUCTION}

The technical problem of designing the optimal shapes of the pump impeller using CFD, i.e. coupling of optimization methods with CFD tools, has been a subject of many articles during the last three decades [Antaki 1995, Miyauchi 2004, Wu 2008, Fan 2011, Zhu 2015, Suh 2019]. A basic shape optimization cycle utilizing CFD could be described by the diagram shown in Fig. 1, where a part called Optimization Environment is in this research substituted by above mentioned optimization method: Particle Swarm Optimization (PSO).

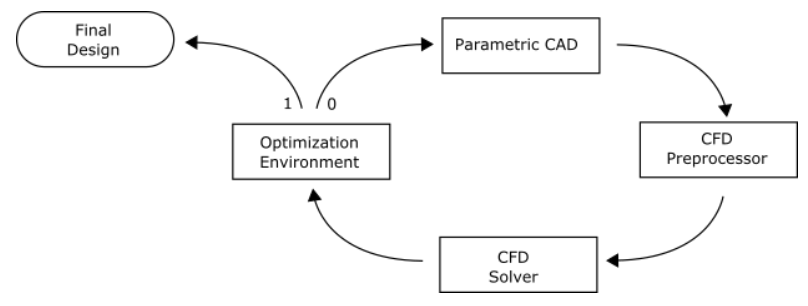

Figure 1. Shape optimization process [Engisoft 2019]

It must be noted that the proper shape of the centrifugal pump impeller and hence successful design strongly depends on several key qualities, namely achieving the required head $\mathrm{H}(\mathrm{m})$, hydraulic efficiency $\eta_{H}(\%)$ and avoiding cavitation, which is often evaluated on the basis of the lowest value of a static pressure on a surface of a blade $p_{\text {stat }}(\mathrm{Pa})$. To successfully combine and fully exploit these qualities, it is recommended to utilize multiobjective optimization and Pareto principles.

A new tool for automatic shape optimization of hydraulic machine impeller, which combines Multi-objective PSO (sec. 2) with various tools from commercial ANSYS CFD package (BladeGen, TurboGrid, CFX) was created for the shape optimization of the impeller of a pump turbine, which can operate as a turbine in a generating mode and as a centrifugal pump in a mode, when excess energy is accumulated in a form of potential energy for further use. The shape optimization is done only for a pump mode of the pump turbine, since the pump mode is the main priority.

\section{MULTI-OBJECTIVE PARTICLE SWARM OPTIMIZATION (MOPSO)}

\subsection{Algorithm form}

The crucial movement of the particles inside the given computational space is captured by following two main equations. First equation is a particle velocity (step size) calculation [Coello 2004]:

$$
\begin{aligned}
v_{i n}= & w \cdot v_{i n}+c_{1} \cdot \operatorname{rand}() \cdot\left(p_{i n}-x_{i n}\right)+ \\
& +c_{2} \cdot \operatorname{Rand}() \cdot\left(\operatorname{rep}_{n}-x_{i n}\right),
\end{aligned}
$$

and the second equation is a particle position computation [Coello 2004]:

$$
x_{i n}=x_{i n}+v_{i n}
$$

where $c_{1}$ and $c_{2}$ are two positive constants; $w$ is inertia weight, rand () and $\operatorname{Rand}()$ are two random vectors from range $(0,1)$; $v_{i n}$ is particle velocity (particle step size) of the $\overline{l-t h}$ particle; $x_{i n}$ is a current position of the $\overline{l-t h}$ particle; $p_{i n}$ is the best previous position of the $\overline{l-t h}$ particle (personal best); $r e p_{n}$ is a leader particle selected by a roulette wheel selection from an external archive (the main task of the external archive is to store nondominated solutions - see sec. 2.2).

\subsection{Pareto principles}

A certain solution is Pareto dominant (optimal), if no other solution exists, which would improve any of the objective functions without degrading at least one of other objectives functions [Coello 2004].

Fig. 2 shows a graphic evaluation of a two-dimensional problem. A large group of the possible solutions of the problem is strongly noticeable (blue circles), but only red circles are nondominated by others and form a Pareto front, which is deeply utilized in presented shape optimization of the centrifugal pump impeller.

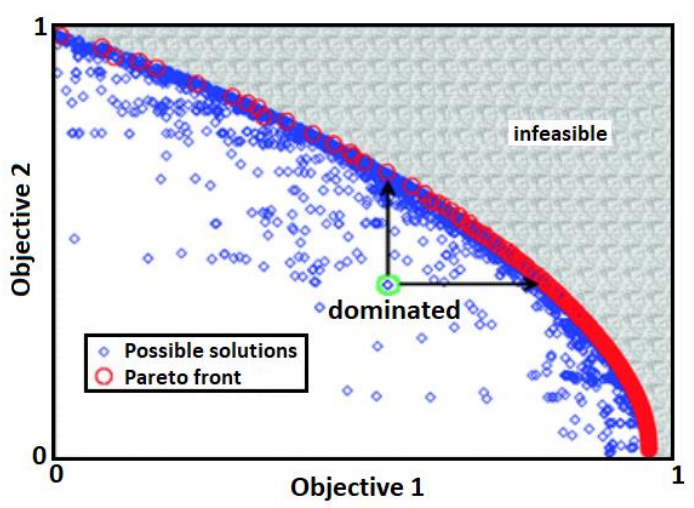

Figure 2. Pareto front (bi-objective optimization) [Sunil Srinivasa 2019] 


\section{SHAPE OPTIMIZATION}

\subsection{Shape optimization problem}

The main goal of presented shape optimization procedure is to create the proper design of the pump impeller, which operates only in pump mode and is based on the requested (given) parameters (Tab. 1).

\begin{tabular}{|c|c|c|}
\hline & Value & Unit \\
\hline Volume flow rate Q & 0.181 & $\mathrm{~m}^{3} / \mathrm{s}$ \\
\hline Pump head H & 34.77 & $\mathrm{~m}$ \\
\hline Shaft speed $\mathbf{n}$ & 1100 & RPM \\
\hline
\end{tabular}

Table 1. Design point of the centrifugal pump (requested parameters)

\subsection{Shape optimization procedure}

The shape optimization procedure is carried out by a code written in a Matlab programming language and compiled within GNU Octave software (license-free software).

The presented shape optimization procedure of the centrifugal pump impeller starts with these prerequisites (green and blue boxes in Fig. 3):

- $\quad$ Requested pump head $\mathrm{H}(\mathrm{m})$ - Tab. 1

- Requested optimal volume flow rate $Q\left(\mathrm{~m}^{3} / \mathrm{s}\right)-T a b .1$

- $\quad$ Requested shaft rotational speed (RPM) - Tab. 1

- Basic impeller properties (blade thickness, number of blades and inlet/outlet points of meridional flow channel)

The shape optimization continues with a basic geometrical outline of the blade of the pump (1D design). This part is based on combination of empirical relations by several authors [Hlbocan 2012, Gulich 2014].

After the initial geometrical layout, the code focuses on handling of following sub-procedures (Fig. 3):

- MOPSO algorithm (sec. 2.1) + objective function evaluation ( $\mathrm{sec} 3.3$ )

- ANSYS BladeGen - impeller modeller (geometry parametrization by Bézier curves)

- ANSYS TurboGrid - mesh generator (hexahedral elements)

- $\quad$ ANSYS CFX - CFD simulations (steady state with only one periodical flow channel)

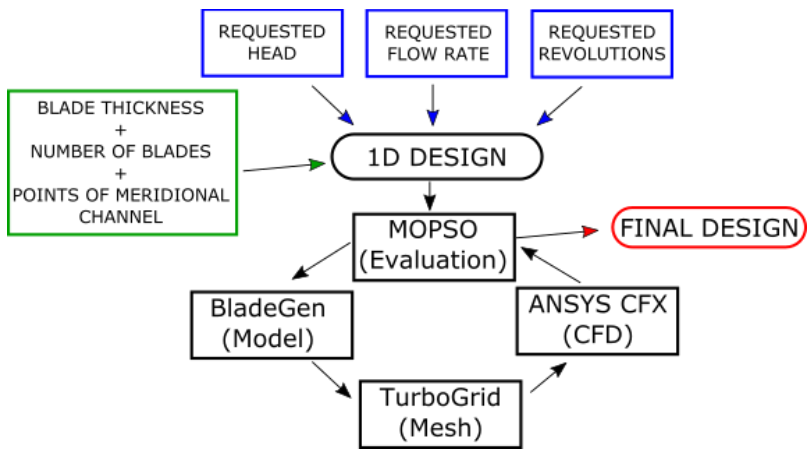

Figure 3. Shape optimization procedure

It must be noted:

- The main outcome of the shape optimization presented in this paper are two pump impellers called Design B and Design C (in more detail in chapter 4).

- Every tool from ANSYS package runs in a so-called batch mode without any graphical user interface. This software mode is handled via text scripts, which enable to run and control these tools directly from the Matlab code created in GNU Octave.
- All main curves that define the meridional flow channel of the pump impeller (hub, shroud, leading edge of the blade) were parametrized by Bézier curves. For a $\beta_{B}$-angle development was utilized linear approach (Design B - chapter 4) and once again Bézier curve (Design C-chapter 4), which created inflection point on parametrized streamline.

- $\quad$ Design B had 22 changing parameters in its parametric model, design $C$ had 28 (due to the different approach in the $\beta_{B}$-angle development).

- Ten particles were used for presented shape optimization (mainly due to limiting aspects of available computational resources). Each design optimization had total number of 25 iterations.

\subsection{Evaluation function}

The key task of the evaluation function $\mathbf{f}$ (Eq. 3) is to select suitable solution (centrifugal pump impeller shape) from the external archive, where the nondominated solutions are stored. This selection is purely based on the user parameter experience. Definition of the multi-objective function combines the requirements on achieved head, shape of efficiency curve (i.e. required efficiency in 3 operating points), avoiding cavitation, preventing unrealistically deformed shapes of impeller discs and blade trailing edge and preventing instability of $\mathrm{H}-\mathrm{Q}$ curve.

$$
\begin{aligned}
f= & w_{H} \cdot\left|1-\frac{H_{o p t}}{H_{\text {req }} / C_{H}}\right|+w_{\eta} \cdot\left(3-\eta_{H,-}-\eta_{H, o p t}-\right. \\
& \left.-\eta_{H,+}\right)+w_{S} \cdot\left(1-C_{\text {press }}\right)+\text { pen }_{\text {shr }}+\text { pen }_{\text {hub }}+ \\
& + \text { pen }_{\text {theta }}+\text { pen }_{H Q},
\end{aligned}
$$

where $H_{\text {opt }}(\mathrm{m})$ is pump head acquired from CFD in optimal flow rate; $\eta_{H,-}(-)$ is hydraulic efficiency acquired from CFD for operating point with flow rate 25 (\%) lower than the design one; $\eta_{H, o p t}(-)$ is hydraulic efficiency acquired from CFD in the design point; $\eta_{H,+}(-)$ is the hydraulic efficiency acquired from for operating point with flow rate 25 (\%) higher than the design one. The rest of the variables is described in a following text: $w_{H}, w_{\eta}$, $w_{S}(-)$ are operational weights, which are prescribed by the designer; $C_{H}(-)$ is constant, which considers hydraulic losses in a complete pump turbine system; $C_{\text {press }}(-)$ is a variable, which focuses on a static pressure on the blade of the impeller (risk of cavitation is evaluated from static pressure value); $p e n_{s h r}$, pen $_{h u b}(-)$ are penalties, which penalize deformed shapes of pump shroud and hub; pen theta $(-)$ is penalty, which penalizes deformed shapes of trailing edge of a blade; pen $_{H Q}(-)$ is penalty, which penalizes a $\mathrm{H}-\mathrm{Q}$ instability (= at a partload).

It must be noted:

- Operational weights were only connected with the main pump qualities (head, efficiency, cavitation). For both optimized designs (see in more detail in chapter 4) were chosen based on the experience to favour designs with the pump head in the close proximity of the requested design point (see Tab. 1). In numbers: $w_{H}=6, w_{\eta}=2, w_{s}=3$. Other combinations of the operational weights were not investigated.

- Penalties were constructed without operational weights. All penalties lied in similar limited range.

\section{RESULTS}

Three different impellers are in detail compared in following text. Design A (red colour) is based on the early stage of the optimization procedure and a quasi-potential flow approach 
[Pochyly 2016, Pochyly 2019], design B (blue colour) and design C (green colour) are the primary outcomes of the optimization procedure briefly described in sections above.

\section{$4.1 B_{B}$-angle definition}

Fundamental shape of the blade of the pump impeller is strongly founded on a $\beta_{B}$-angle development from the leading to trailing edge of the blade.
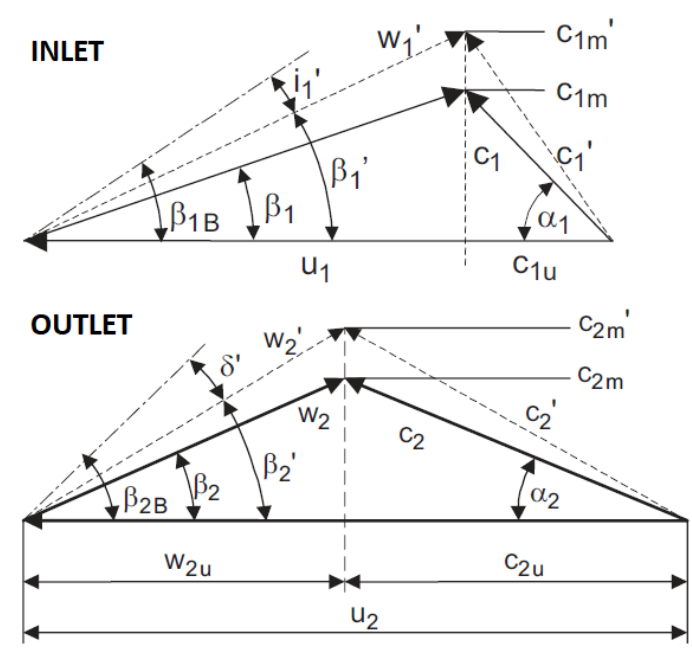

Figure 4. $\beta_{B}$-angle definition [Gulich 2014]

$\beta_{B}$-angle is based on velocity triangles (in Fig. 4 are highlighted at the inlet and outlet of the impeller), where [Gulich 2014]:

- $\quad c(\mathrm{~m} / \mathrm{s})$ are absolute velocities

- $\quad w(\mathrm{~m} / \mathrm{s})$ are relative velocities

- $u(\mathrm{~m} / \mathrm{s})$ are circumferential velocities

- index $u$ represents circumferential velocity components

- index $m$ represents meridional velocity components

- $\alpha\left({ }^{\circ}\right)$ are angles in absolute reference frame

- quantities with raised stroke are affected by a blade blockage due to a finite thickness of the blade

- $i\left({ }^{\circ}\right)$ is an incidence, which characterizes difference between the real flow and the blade inlet angle $\beta_{1 \mathrm{~B}}$

- $\delta\left({ }^{\circ}\right)$ is a deviation angle, which characterizes difference between the real flow and the blade outlet angle $\beta_{2 \mathrm{~B}}$.

\subsection{Pump head and hydraulic efficiency definition}

Two main performance quantities were evaluated in section 4.5 . First, the pump head $\mathrm{H}(\mathrm{m})$, which is defined [Pochyly 2019]:

$$
H=\frac{p_{\text {total }, o u t}-p_{\text {total,in }}}{\rho \cdot g}
$$

where $p_{\text {total,in }}(\mathrm{Pa})$ is a total pressure at evaluation inlet, $p_{\text {total,out }}(\mathrm{Pa})$ is a total pressure at evaluation outlet, $\rho\left(\mathrm{kg} / \mathrm{m}^{3}\right)$ is a water density, $g\left(\mathrm{~m} / \mathrm{s}^{2}\right)$ is a gravitational acceleration.

And second, the hydraulic efficiency $\eta_{H}(\%)$, which is defined [Pochyly 2019]:

$$
\eta_{H}=\frac{\left(p_{\text {total,out }}-p_{\text {total,in }}\right) \cdot Q}{2 \cdot \pi \cdot n \cdot M_{k}} \cdot 100,
$$

where $Q\left(\mathrm{~m}^{3} / \mathrm{s}\right)$ is the volume flow rate, $n\left(\mathrm{~s}^{-1}\right)$ is the shaft rotational speed, $M_{k}(\mathrm{Nm})$ is a torque.

\subsection{CFD simulations}

Unsteady (URANS) simulations were utilized for the purpose of data correlation between the optimized designs. Each impeller was inserted into the complete pump turbine system, which is demonstrated in Fig. 5.

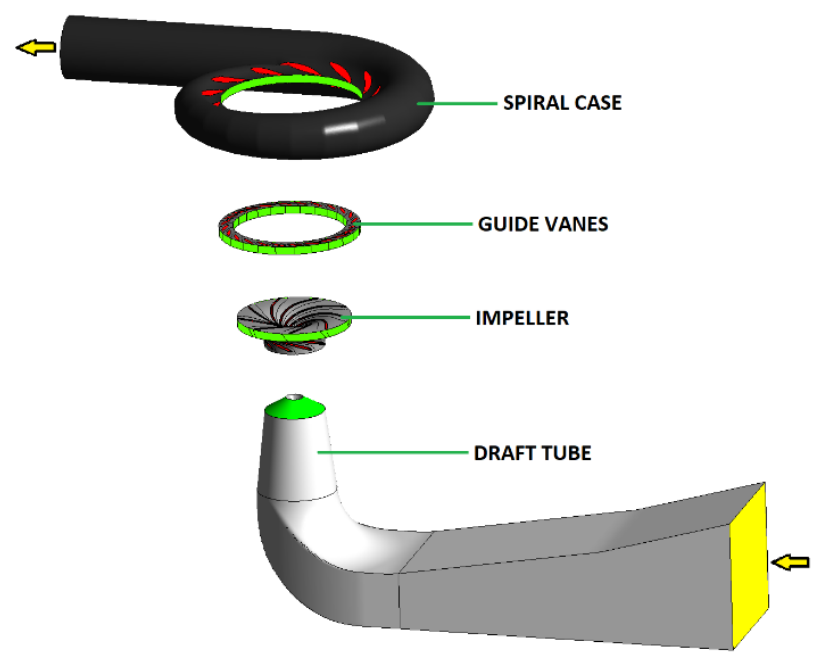

Figure 5. Computational domains

The computational mesh of all pump turbine flow domains such as guide vanes, all optimized impellers and the draft tube were built in ICEM CFD and TurboGrid tool as fully hexahedral (Fig. 6). The computational mesh of the spiral case (pump volute) was created in ANSYS Workbench meshing as tetrahedral with high-resolution prismatic layers near solid walls of the domain (Fig. 6).

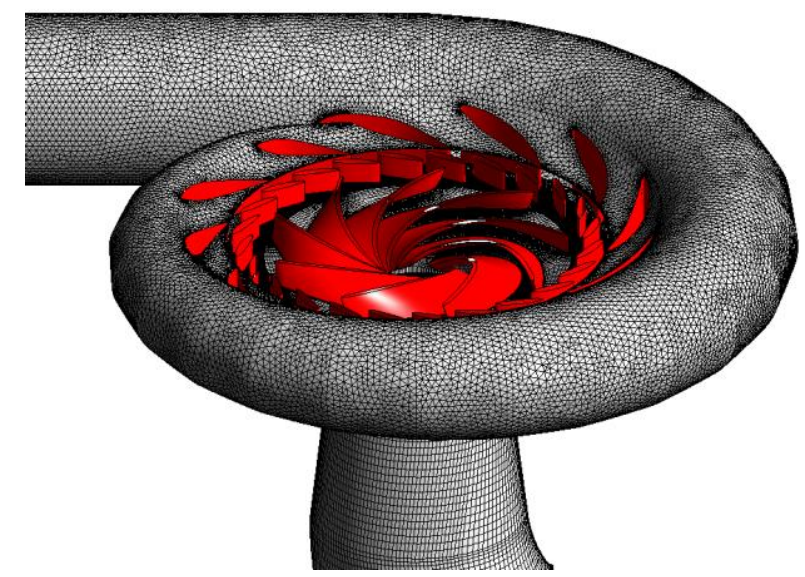

Figure 6. Sample of the computational mesh

All presented CFD simulations of the complete pump turbine system were performed by commercial ANSYS CFX tool with a standard $k-\varepsilon$ model of turbulence. Model of turbulence was chosen for its robust computational qualities. A timestep of each calculation corresponded approximately to $3\left({ }^{\circ}\right)$ of the pump impeller revolution, with 5 inner iterations. Boundary conditions were set as follows (Fig. 5 - yellow arrows): for inlet boundary condition it was zero relative static pressure and for outlet boundary condition it was mass flow rate, which was derived from values of the volume flow rate using water density $\rho\left(\mathrm{kg} / \mathrm{m}^{3}\right)$ - default value of the water density in ANSYS CFX is set to $997\left(\mathrm{~kg} / \mathrm{m}^{3}\right)$. Steady state RANS calculations with frozen rotor type of interface between stationary and rotational parts of the pump turbine served for transient simulation initialization.

\subsection{Geometrical comparison}

All three optimized impellers are captured in Fig. 7., where one blade of the pump impeller is drawn over one another to expose important differences. Complete overall view of all three pump impellers is shown in Fig. 11.

The design $A$ has the longest blades with a lying shape characteristic. The design $B$ has on the other hand the shortest length of the blade. In the middle (between designs A and B) lies 
green design $\mathrm{C}$ with a blade length compromise and with a significant leading edge curvature from the shroud to the hub.

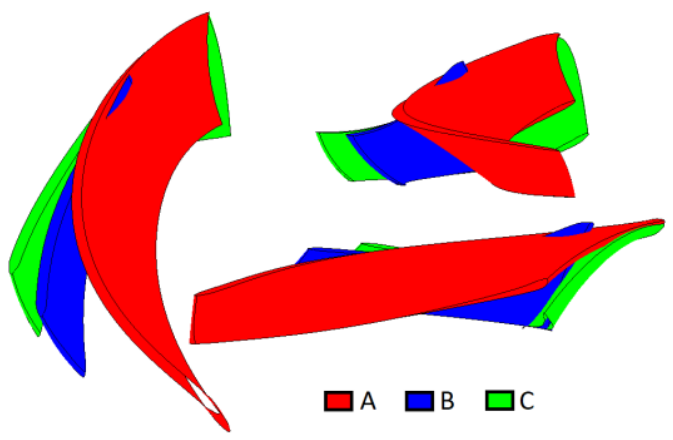

Figure 7. Blade comparison [Pochyly 2019]

It must be noted that length of the blades has a crucial role on proper water guiding in the blade channel. Short blades must accommodate higher static pressure values on the lower blade area. Long-shaped blades cause higher hydraulic losses due to larger wetted area interaction between blade walls and water.

The blades of the optimized impellers could be compared also from the blade angle perspective. The $\beta_{B}$-angle development along the blade length plays crucial role in energy transfer from blade to flowing water. This paper compares three $\beta_{B}$-angle developments: quasi-potential foundation in the design $A$, the linear approach of the design $B$ and the inflection point approach utilized in the design $C$ (this method is founded on [Gulich 2014]). They are captured for three streamlines (close to pump shroud, in the middle and close to hub) in Figs. 8-10.

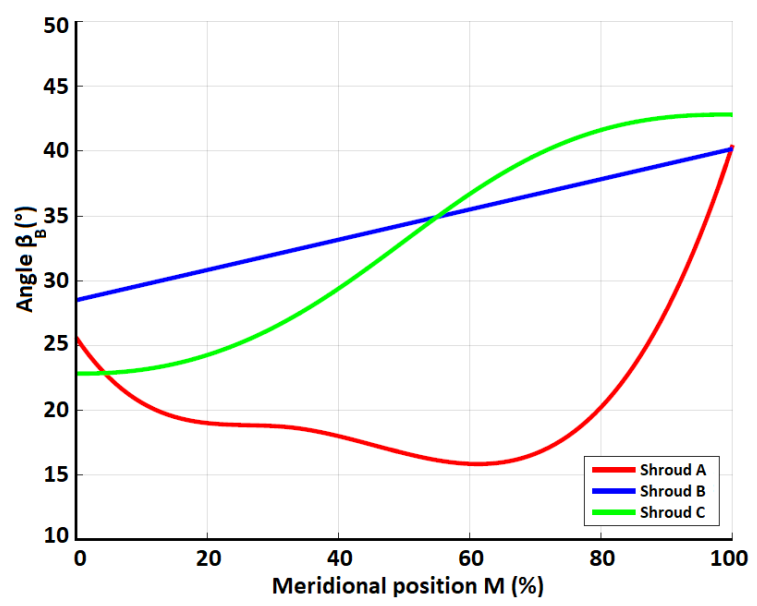

Figure 8. $\beta_{\mathrm{B}}$-angle development on shroud

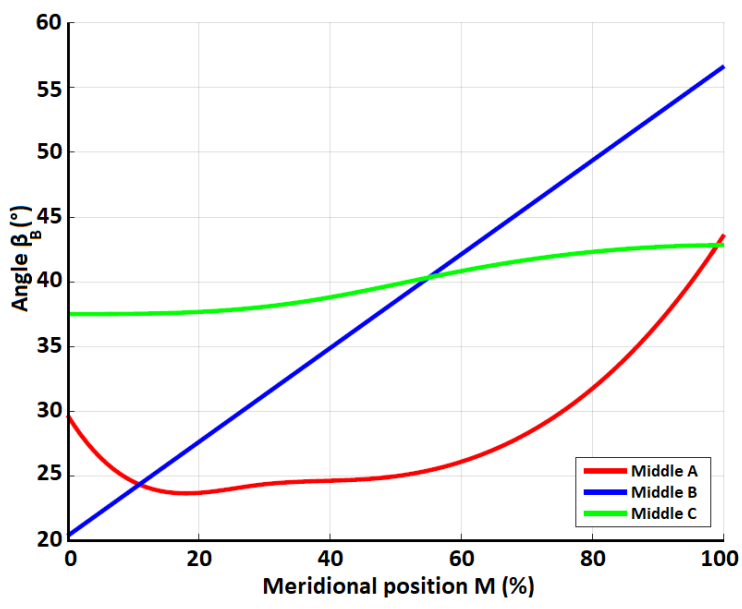

Figure 9. $\beta_{\mathrm{B}}$-angle development on middle streamline

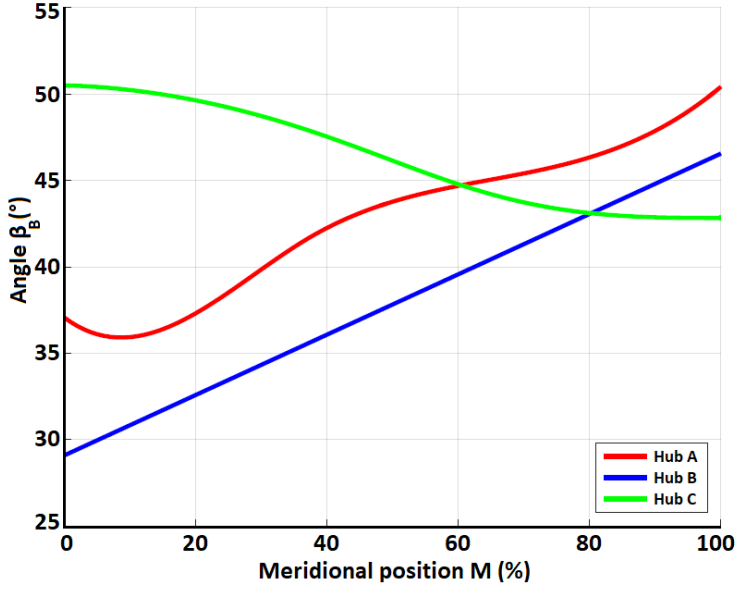

Figure 10. $\beta_{\mathrm{B}}$-angle development on hub

Red design $A$ has the most complicated $\beta_{B}$-angle development among all three optimized pump impellers. This development was strongly based on the main output from the in-house shape optimization software utilizing quasi-potential flow approach [Pochyly 2016, Pochyly 2019]. The $\beta_{B}$-angle development near the shroud and on the middle streamline almost mimics quadratic function, with the lowest values of $\beta_{B}$-angle located around the middle part of the blade.

Design B exploited a pure linear concept of the blade modelling. This approach is the simplest and utilized only inlet/outlet values of $\beta_{B}$-angle, therefore significantly reducing requirements on geometry parametrization.

Design $\mathrm{C}$ was built according to recommendation in [Gulich 2014], where the inflection point in the $\beta_{B}$-angle development is outlined. Mentioned inflection point provided mild $\beta_{B}$-angle change near the pump inlet (reducing the blade loading [Gulich 2014]) and also near the outlet to allow for convenient blade trimming [Gulich 2014].

A
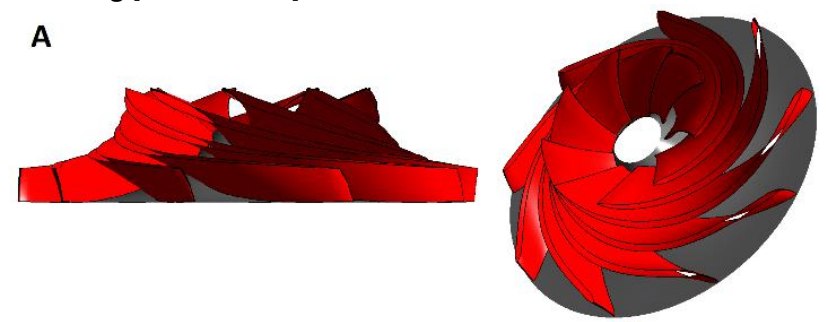

B
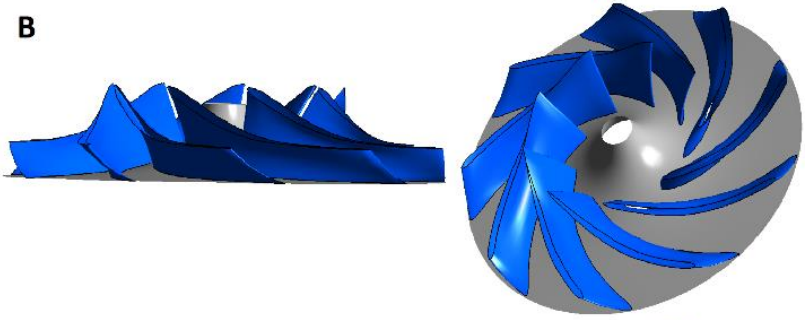

C
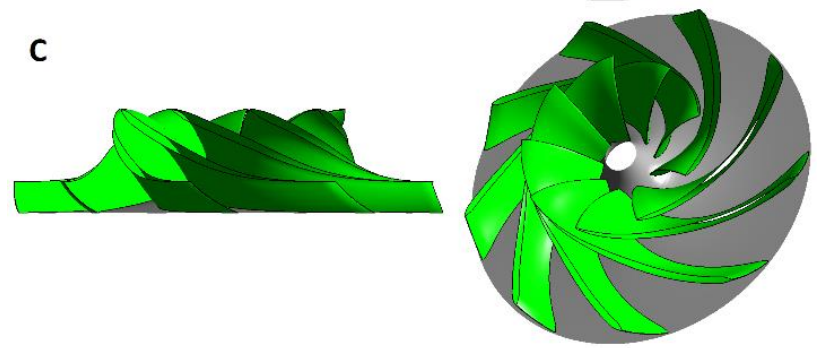

Figure 11. Pump impeller designs [Pochyly 2019]

\subsection{Performance characteristics comparison}

Two main performance characteristics were obtained from URANS simulations for all optimized impeller designs: 
$\mathrm{H}-\mathrm{Q}$ dependency (Fig. 12) and $\eta_{\mathrm{H}-\mathrm{Q}}$ dependency (Fig. 13). These principal curves were plotted only for one fixed guide vanes opening $-\mathrm{a}_{0}=20 \mathrm{~mm}$.

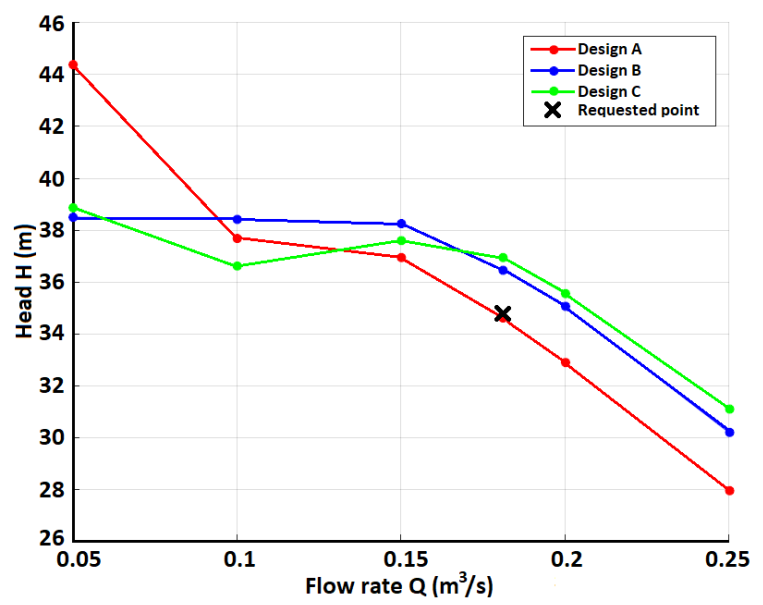

Figure 12. $\mathrm{H}-\mathrm{Q}$ dependency

It is noticeable that only red design A (Fig. 12) managed to fulfil requested design point defined by Tab. 1 . The blue design $B$ and green design $C$ exceeded the requested point by approximately $4(\%)$ and $5(\%)$, respectively.

Significant pump head overestimations/underestimations are observed mainly in region defined by volume flow rate $Q<0.15\left(\mathrm{~m}^{3} / \mathrm{s}\right)$. This unfavourable fact is caused by a very complex and turbulent flow in partial load regimes of the pump turbine. Standard k- $\varepsilon$ model of turbulence chosen for URANS simulations was unable to capture properly such complicated flow problem.

The second observed performance characteristic was $\eta_{H^{-}} \mathrm{Q}$ dependency (Fig. 13). Green design $C$ outperformed the other two impellers in terms of hydraulic efficiency values in close vicinity of the requested pump optimum, which is marked by a black arrow in Fig. 13. A difference in the optimal flow rate $\left(\mathrm{Q}=0.181\left(\mathrm{~m}^{3} / \mathrm{s}\right)\right)$ between the design $\mathrm{A}$ (red colour) and design $\mathrm{C}$ (green colour) is approximately 5 (\%).

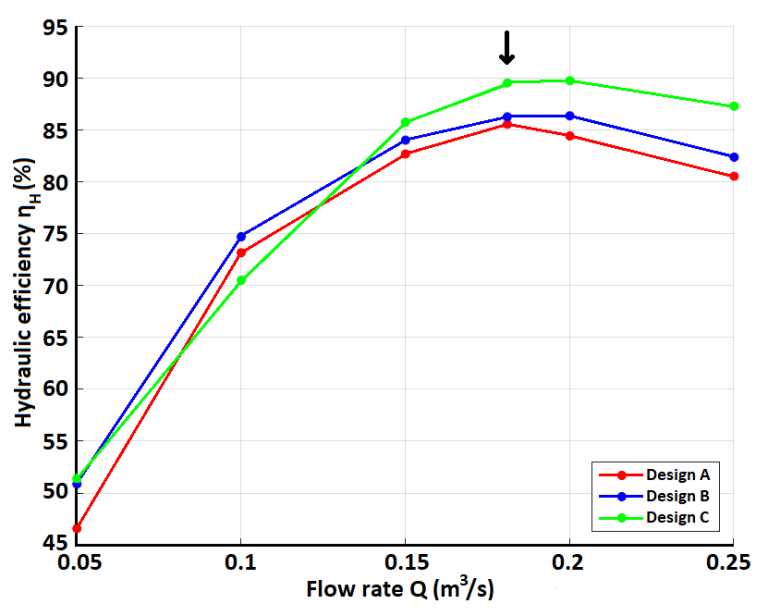

Figure 13. $\eta_{H^{-}} \mathrm{Q}$ dependency

\subsection{Flow patterns comparison}

Figure 14 shows a basic comparison of the optimized pump impellers from the meridional point of view. Figure displays average static pressure within the flow passage of the pump impeller. The leading edge of the blade is highlighted for each impeller by a thick red curve. The values of the average static pressure were clipped in the same interval for all three designs (3.0e5, -7.0e4) (Pa).

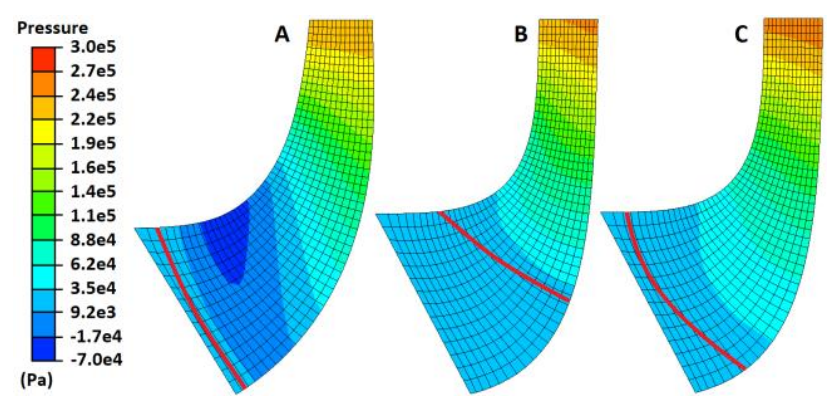

Figure 14. Meridional flow channel - average static pressure; optimal flow rate

It is obvious from Fig. 14 that only design A has a significant average static pressure drop in observable meridional point of view. This pressure drop is located near the leading edge of the blade in a region close to the shroud. This phenomenon is caused by substantial $\beta_{B}$-angle change (decrease) near the leading edge on all three modelled streamlines.

A sudden static pressure drop manifestation must be avoided in a process of designing proper shapes of pump impellers to avoid cavitation and its negative effects.

Figs. 15-17 capture fluid flow directly on the walls of the blade by plotting surface streamlines in the optimal flow rate of the pump. This graphical representation reveals recirculation passages in designs $A$ and $B$, which are in both cases located on suction side of the blades near the trailing edge of the shroud streamline. The recirculation mainly negatively affects pump head and hydraulic efficiency values.

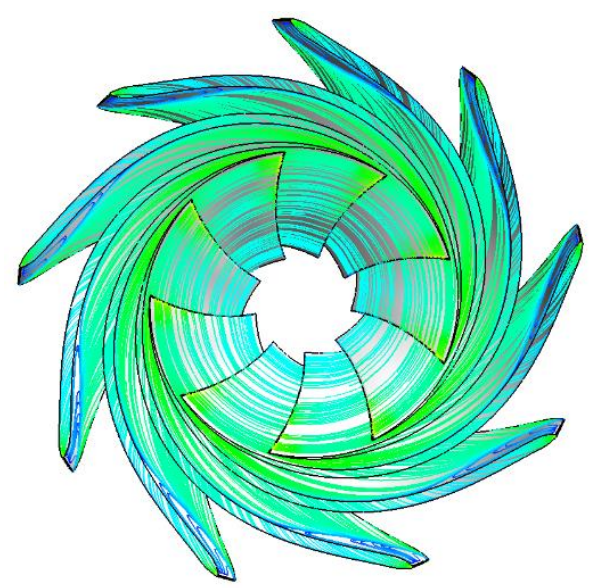

Figure 15. Surface streamlines design A - optimal flow

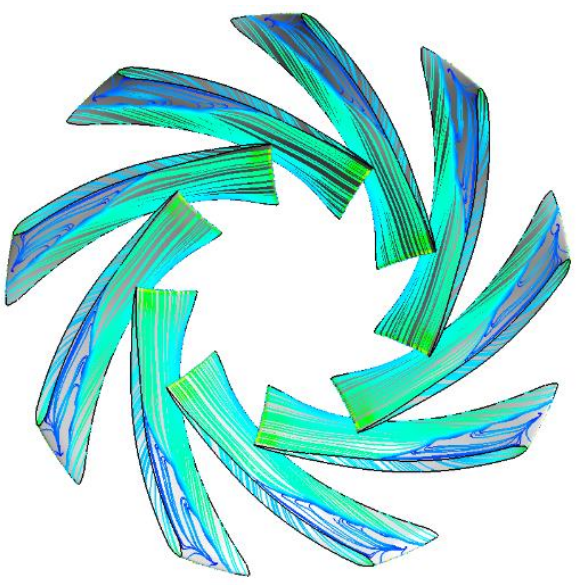

Figure 16. Surface streamlines design B - optimal flow 


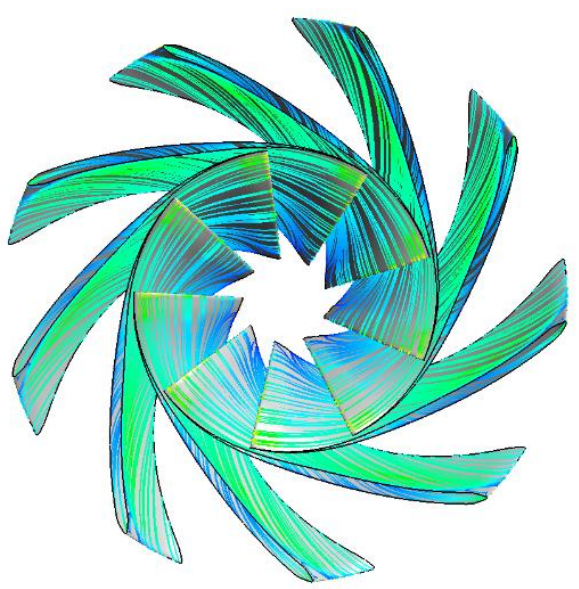

Figure 17. Surface streamlines design C - optimal flow rate

\section{CONCLUSIONS}

A new tool for automatic shape optimization of hydraulic machine impeller, which combines mathematical optimization method (namely Multi-objective PSO) with set of tools from commercial ANSYS CFD package (BladeGen, TurboGrid, CFX) was presented in the paper. The Particle Swarm Optimization method shows its quality mainly thanks to random scattering of individuals over the explored space providing sufficiently global and robust behaviour. However, it must be noted that total computational time and a computational capacity demand increase with large swarms of individuals and a reasonable compromise has to be made between number of swarm individuals and versatility of the optimization procedure.

It is clear from the comparative numerical simulations of the fluid flow inside the passages of the pump turbine that the blades of the radial pump impeller should be as simple as possible. The radically diverse blade shapes show the unfavourable flow patterns inside the impeller, which leads to the lower values of the hydraulic efficiency and possible cavitation manifestation, due to sudden pressure drops inside the flow passages. The moderate development of the $\beta_{B}$-angle near the leading edge of the blade shows to be convenient (on all modelled streamlines) - this fact decreases some portion of the blade loading. It is also recommended to achieve a gradual static pressure change in the meridional point of view (Fig. 14), without unexpected pressure abnormalities.

\section{ACKNOWLEDGMENTS}

The research has been supported by project "Computer Simulations for Effective Low-Emission Energy" funded as project No. CZ.02.1.01/0.0/0.0/16_026/0008392 by Operational Programme Research, Development and Education, Priority axis 1: Strengthening capacity for high-quality research.

\section{REFERENCES}

[Antaki 1995] Antaki, J., et al. Computational Flow Optimization of Rotary Blood Pump Components. Artificial Organs [online]. Oxford, UK: Blackwell Publishing, 1995, vol. 19, no. 3, pp. 608-615 [cit. 2019-29-12]. DOI: 10.1111/j.1525-1594.1995.tb02389.x. ISSN 0160$564 X$.

[Coello 2004] Coello, C.A.C., Pulido, G.T. and Lechuga, M.S. Handling multiple objectives with particle swarm optimization. IEEE Transactions on Evolutionary
Computation, June 2004 , vol. 8 , no. 3, pp. 256-279 ISSN 1089778X.

[Enginsoft 2019] Enginsoft. Shape optimization diagram. 2019 [online], [cit. 2019-29-12]. Available from http://www.enginsoft.com/assets/img/tecnology/cf d/meshmorphing_01.png.

[Fan 2011] Fan, J., et al. Computational fluid dynamic analysis and design optimization of jet pumps. Computers and Fluids [online]. Elsevier, 2011, vol. 46, no. 1, 212-217 [cit. 2019-29-12]. DOI: 10.1016/ j.compfluid.2010.10.024. ISSN 0045-7930.

[Gulich 2014] Gulich, J.F. Centrifugal pumps. Heidelberg: Springer, 2014. ISBN 978-3-642-40113-8.

[Hlbocan 2012] Hlbocan, P. and Varchola, M. Prime Geometry Solution of a Centrifugal Impeller Within a 3D Setting. Procedia Engineering [online]. Elsevier, 2012, vol. 39, pp. 197-203 [cit. 2019-29-12]. DOI: 10.1016/j.proeng.2012.07.025. 9781627486026. ISSN 1877-7058.

[Miyauchi 2004] Miyauchi, S., et al. Optimization of Meridional Flow Channel Design of Pump Impeller. International Journal of Rotating Machinery [online]. Hindawi Publishing Corporation, 2004, vol. 10, no. 2, [cit. 2019-29-12]. DOI: 10.1155/S1023621X04000120. ISSN 1023-621X.

[Pochyly 2016] Pochyly, F. and Stejskal, J. Rotational Flow in Centrifugal Pump Meridian Using Curvilinear Coordinates. Journal of Fluid Engineering Transactions of the ASME, 2016, vol. 138, no. 8, pp. 1765-1770. ISSN: 0098-2202.

[Pochyly 2019] Pochyly, F., Rudolf, P., Stefan, D., Moravec, P., Stejskal, J. and Skotak, A. Design of a pump-turbine using a quasi-potential flow approach, mathematical optimization and CFD [online]. IOP Conference Series: Earth and Environmental Science. March 2019, vol. 240, [cit. 2019-29-12]. Available from https://iopscience.iop.org/article/10.1088/17551315/240/7/072043.

[Suh 2019] Suh, J., et al. Multi-objective optimization of a high efficiency and suction performance for mixed-flow pump impeller. Engineering Applications of Computational Fluid Mechanics [online]. Taylor \& Francis, 2019, vol. 13, no.1, pp. 744-762 [cit. 201929-12]. DOI: 10.1080/19942060.2019.1643408. ISSN 1994-2060.

[Sunil Srinivasa 2019] Enginsoft. Multi-objective Reinforcement Learning [online], [cit. 2019-29-12]. Available from https://sunil-s.github.io/assets/images/2016-12-10MORL/ParetoFront.PNG.

[Wu 2008] Wu, K.-H, Lin, B.-J. and Hung, C.-I. Novel Design of Centrifugal Pump Impellers Using Generated Machining Method and CFD. Engineering Applications of Computational Fluid Mechanics [online]. Taylor \& Francis, 2008, vol. 2, no. 2, pp. 195207 [cit. 2019-29-12]. DOI: 10.1080/ 19942060.2008.11015221. ISSN 1994-2060. 
[Zhu 2015] Zhu, B., et al. Optimization design of a reversible vol. 81, pp. 366-376 [cit. 2019-29-12]. DOI: 10.1016/ pump-turbine runner with high efficiency and stability. Renewable Energy [online]. Elsevier, 2015,

\section{CONTACTS}

doc. Ing. Pavel Rudolf Ph.D.

Brno University of Technology, V. Kaplan Department of Fluid Engineering Technická 2896/2, 61669 Brno-Královo Pole, Czech Republic rudolf@fme.vutbr.cz

Ing. Prokop Moravec

Brno University of Technology, V. Kaplan Department of Fluid Engineering Technická 2896/2, 61669 Brno-Královo Pole, Czech Republic

115861@vutbr.cz 